\title{
A single-arm, multicenter, phase II clinical study of tislelizumab plus albumin-bound paclitaxel/cisplatin as neoadjuvant therapy for borderline resectable esophageal squamous cell carcinoma
}

\author{
Xiaodong $\mathrm{Li}^{1 \#}$, Congcong Xu ${ }^{1 \#}$, Hongbin Qiu ${ }^{1 \#}$, Dong Chen ${ }^{1}$, Kanghao Zhu ${ }^{2}$, Bo Zhang ${ }^{1}$, Jian Zhang ${ }^{1}$, \\ Anyi Xu ${ }^{1}$, Chunguo Wang ${ }^{1}$, Jianfei Shen ${ }^{1}$ \\ ${ }^{1}$ Key Laboratory of Minimally Invasive Techniques \& Rapid Rehabilitation of Digestive System Tumors of Zhejiang Province, Department of \\ Cardiothoracic Surgery, Taizhou Hospital of Zhejiang Province Affiliated to Wenzhou Medical University, Linhai, China; ${ }^{2}$ Key Laboratory of \\ Minimally Invasive Techniques \& Rapid Rehabilitation of Digestive System Tumors of Zhejiang Province, Department of Cardiothoracic Surgery, \\ Taizhou Hospital, Zhejiang University, Linhai, China \\ \#These authors contributed equally to this work. \\ Correspondence to: Chunguo Wang; Jianfei Shen. Key Laboratory of Minimally Invasive Techniques \& Rapid Rehabilitation of Digestive System \\ Tumors of Zhejiang Province, Department of Cardiothoracic Surgery, Taizhou Hospital of Zhejiang Province Affiliated to Wenzhou Medical \\ University, Linhai 317000, China. Email: wangchg@enzemed.com; jianfei051@163.com.
}

Background: Esophageal cancer responds poorly to conventional radiotherapy, chemotherapy, and/or surgery. Immunotherapy works by boosting the body's immune system, and preoperative immunotherapy combined with chemotherapy may increase the survival rate of patients with esophageal cancer. Here we further explore immunotherapy's role in treating borderline resectable (BR) esophageal squamous cell carcinoma (ESCC) by combining immunotherapy with chemotherapy.

Methods: In this multicenter, randomized controlled study of preoperative immunotherapy plus chemotherapy for BR ESCC, immunotherapy plus chemotherapy [i.e., tislelizumab plus albumin-bound paclitaxel (ABP)/cisplatin] will be given according to the inclusion and exclusion criteria. Patients are to be observed and recorded for various indicators, the follow-up visits are standardized, and a database is to be established for the statistical analysis, with an attempt to clarify the value of preoperative immunotherapy plus chemotherapy in improving the survival of patients with BR ESCC. The primary endpoints are diseasefree survival (DFS), major pathologic response (MPR), and pathologic complete response (pCR). The secondary endpoints include the objective response rate (ORR) and overall survival (OS) in subjects with PD-L1 expression levels of $<1 \%, \geq 1 \%, \geq 20 \%$, and $\geq 50 \%$.

Discussion: The role of preoperative concurrent immunotherapy plus chemotherapy in improving the survival rates of patients with BR ESCC will be explored in this study. Given that the 5-year survival rate of BR ESCC is $10 \%$, we hope that a reasonable immunotherapy plus chemotherapy regimen with higher efficacy and lower toxicity will further increase the pCR.

Trial Registration: Chinese Clinical Trial Registry Identifier: ChiCTR2100051514.

Keywords: Tislelizumab; borderline resectable esophageal squamous cell carcinoma (BR ESCC); neoadjuvant immunotherapy; albumin-bound paclitaxel (ABP); cisplatin

Submitted Sep 28, 2021. Accepted for publication Jan 27, 2022.

doi: 10.21037/atm-21-6931

View this article at: https://dx.doi.org/10.21037/atm-21-6931 


\section{Introduction}

Esophageal cancer is one of the common malignant tumors in the digestive system. According to the global cancer statistics from 2018, the incidence and mortality of esophageal cancer continue to rise in some regions $(1,2)$. In China, esophageal squamous cell carcinoma (ESCC) accounts for more than $95 \%$ of esophageal cancer patients. Clinicopathological and epidemiological studies have shown that the main risk factors for ESCC in areas with a high incidence of esophageal cancer in China are $\mathrm{N}$-nitrosamines and their precursors, as well as some fungi and their toxins. ESCC is insidious in onset, with atypical symptoms and high invasiveness (1-7). Esophageal cancer is generally treated with a multidisciplinary strategy, including surgery, radiotherapy, and chemotherapy. The individual treatment modality mainly depends on the disease stage, tumor location, and the patient's general condition. Surgery alone is associated with poor prognosis, with a 5 -year survival of less than $25 \%$ (3). Therefore, the treatment of esophageal cancer has been a hot research topic in recent years, and notable progress has been made in neoadjuvant therapy and immunotherapy.

In recent years, many studies have demonstrated the encouraging efficacy of postoperative adjuvant immunotherapy in treating advanced or locally advanced esophageal cancer (8-16). However, it is still uncertain whether immunotherapy can change the way esophageal cancer is treated, especially whether it can ultimately improve long-term survival. Therefore, the efficacy and safety of preoperative immunotherapy for esophageal cancer need to be further clarified. In clinical settings, if an esophageal cancer patient is identified with a more advanced disease (e.g., T4N2) at their first visit, preoperative neoadjuvant therapy may be offered. More advanced tumors present a higher risk of recurrence. Preoperative treatment may dramatically "downsize" or shrink the tumor, possibly improving the prognosis. The 2020 Esophageal Cancer Treatment Guidelines (17) introduced a new concept, borderline resectable (BR) esophageal cancer, which refers to a radiologically unspecified cT4b stage esophageal cancer suspected to involve the surrounding organs. Since BR esophageal cancer is often in a more advanced stage, preoperative neoadjuvant therapy is required to shrink the tumor and enable surgical treatment. Previous studies have reported neoadjuvant therapy with drugs such as pembrolizumab for locally advanced ESCC $(5,17)$, while the role of immunotherapy with tislelizumab in treating BR ESCC remains unclear.

Chemotherapy, as a systemic adjuvant treatment for cancer, can effectively kill tumor cells and control disease progression. In contrast, immunotherapy combined with chemotherapy can reverse immunosuppression in the advanced stages, improve cross-presentation of tumor antigens, promote proliferation of killer $\mathrm{T}$ cells (and increase their cancer-killing ability), and slow down the development of drug resistance in tumor cells. The combinations of immunotherapy with chemotherapy and surgery may provide new survival benefits for patients. As a new treatment paradigm, perioperative immunotherapy plus neoadjuvant radio-chemotherapy has been explored in patients with locally advanced esophageal cancer and has shown encouraging results. In a phase II trial of preoperative chemoradiotherapy and pembrolizumab for locally advanced ESCC, the pathologic complete response (pCR) rate reached $46.1 \%$ in the combination group, and there was a trend toward better disease-free survival (DFS) in the pCR group ( $\mathrm{n}=12)$ compared with the non-pCR group $(\mathrm{n}=14)(\mathrm{HR}=0.33 ; \mathrm{P}=0.1)(18)$. In the phase II feasibility trial of neoadjuvant chemoradiotherapy combined with atezolizumab for resectable esophageal adenocarcinoma (the PERFECT trial), the pCR rate was 39\% (9/23) in the combination group (19), which was higher than that (23\%) in the CROSS study. Tislelizumab, a humanized IgG4 antiPD-1 monoclonal antibody, was approved for marketing by China's National Medical Products Administration (NMPA) on December 26, 2019. Tislelizumab has been approved for a variety of indications, including relapsed or refractory classical Hodgkin's lymphoma, locally advanced or metastatic uroepithelial carcinoma, unresectable locally advanced or metastatic non-small cell lung cancer, and hepatocellular carcinoma. A randomized, placebo-controlled, doubleblind phase III study (RATIONALE 302) (6) evaluated the efficacy and safety of tislelizumab vs. paclitaxel, docetaxel, or irinotecan as a first-line treatment in patients with advanced recurrent or metastatic ESCC. It was found that, compared to chemotherapy, the tislelizumab group had a significantly longer median survival (8.6 vs. 6.3 months), a significantly lower risk of death (decreased by $30 \%$ ), a higher objective response rate (ORR) (20.3\% vs. 9.8\%), and a longer duration of response (DOR) (7.1 vs. 4.0 months). The RATIONALE 302 study suggested that tislelizumab had a manageable safety profile and promising clinical efficacy in advanced/metastatic esophageal cancer that had failed first-line standard therapy. Here we further conducted a more precise large-scale study investigate of the role of tislelizumab in the neoadjuvant treatment of BR esophageal cancer (6).

We present the following article in accordance with 


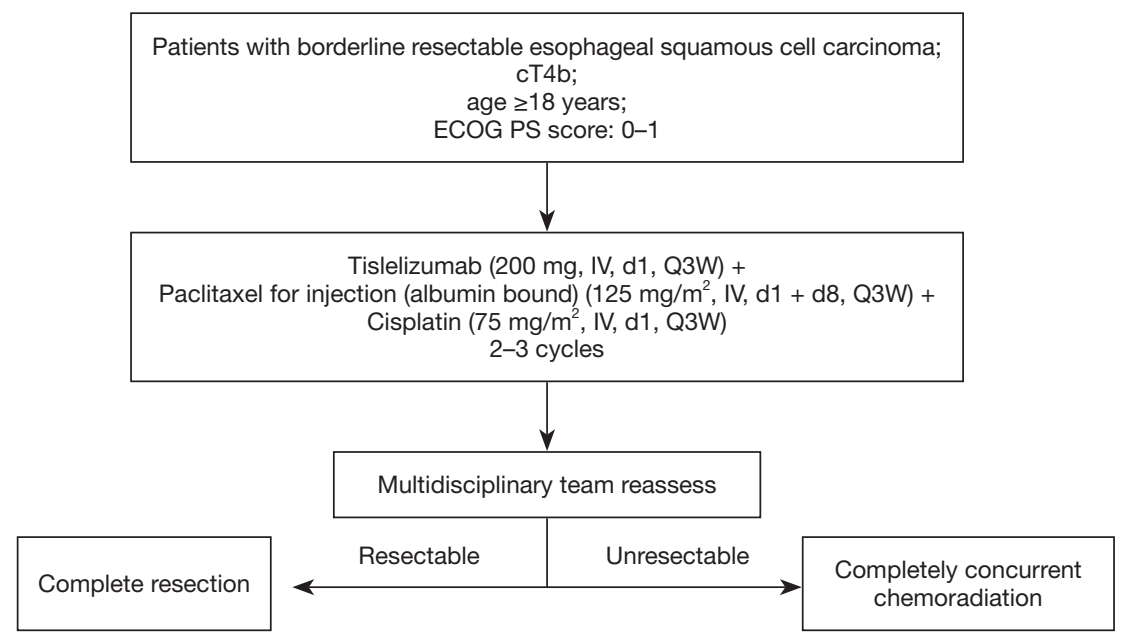

Figure 1 Flow chart of the study design. ECOG PS, Eastern Cooperative Oncology Group performance status.

the SPIRIT reporting checklist (available at https://atm. amegroups.com/article/view/10.21037/atm-21-6931/rc).

\section{Methods}

\section{Study design}

In this single-arm, multicenter, phase II trial, 50 cases are expected to be enrolled. Tislelizumab plus albumin-bound paclitaxel $(\mathrm{ABP}) /$ cisplatin as neoadjuvant therapy will be offered for 2-3 cycles, and surgery will be performed 46 weeks after the last cycle of neoadjuvant immunotherapy combined with chemotherapy, as shown in Figure 1. One to 2 cycle of adjuvant immunotherapy combined with chemotherapy with the above regimen will be administered after surgery until four cycles, and tislelizumab therapy will be maintained for 1 year after the end of adjuvant immunotherapy with chemotherapy. A database will be created, and all patients will be followed up.

\section{Identify the BR ESCC}

For the diagnosis of BR ESCC, tracheobronchial invasion is determined by biopsy results and naked eye observation of rigid capsules or depressions confirmed by bronchoscopy and CT. This study defines BR ESCC as tumor invasion of adjacent organs suspected but not clearly diagnosed as c T4b disease.

\section{Data analysis and processing}

The full analysis set (FAS) will include all subjects who meet the inclusion criteria and have undergone at least one postbaseline outcome assessment. Statistical analyses will be performed using SAS version 9.1 (SAS Institute Inc., Cary, NC, USA) or later. Most of the analyses in this study are descriptive. Summary statistics will be provided. Continuous variables will include numbers, means, medians, standard deviations, and minimum and maximum values. Categorical variables will consist of frequencies and percentages for each category. Survivals will be analyzed using the Kaplan-Meier (log-rank) test. The descriptive summary statistics include numbers, means, standard deviations, medians, ranges, and minimum and maximum values. The FAS-based descriptive statistics will be used to summarize the demographic data and baseline clinical features (including age, sex, ethnicity, height, weight, pathological types, lymph node status, clinical stages, WHO physical status, and laboratory test results).

\section{Drug combinations and specific treatment regimen}

Tislelizumab (200 mg, IV, d1, Q3W), combined with ABP $\left(125 \mathrm{mg} / \mathrm{m}^{2}, \mathrm{IV}, \mathrm{d} 1+\mathrm{d} 8, \mathrm{Q} 3 \mathrm{~W}\right)$ and cisplatin $\left(75 \mathrm{mg} / \mathrm{m}^{2}\right.$, IV, d1, Q3W), will be administered. Adequate hydration will be required before and after cisplatin use (see below for details).

\section{Pretreatment}

Antiemetic prophylaxis for chemotherapy-induced nausea and vomiting: efforts will be made to prevent cisplatininduced acute and delayed nausea and vomiting. For 
acute vomiting (day 1): a 5-HT3 receptor antagonist or dexamethasone $20 \mathrm{mg}$ (oral or IV over 5-15 minutes) will be administered within 1 hour before chemotherapy; for delayed vomiting (day 2-4): antiemetic prophylaxis will be initiated on the morning of chemotherapy day 2 , twice daily for 3 days. DXM $8 \mathrm{mg}$ bid + metoclopramide $20 \mathrm{mg}$ bid for 3 days is recommended. A 5-HT3 receptor antagonist could be used instead of metoclopramide.

\section{Hydration in cisplatin-based therapy}

Pre-hydration: more than $2 \mathrm{~L}$ of fluid will be taken orally 1 day before chemotherapy. Intravenous fluid therapy (1,000 $\mathrm{mL}$ of saline or glucose solution) will be offered on the same day before high-dose cisplatin chemotherapy, and then high-dose cisplatin will be added to $500 \mathrm{~mL}$ of normal saline (NS) before intravenous administration.

Post-hydration: 5\% glucose in normal saline (GNS) and $5 \%$ glucose solution (GS) or NS will be given intravenously immediately after the end of cisplatin chemotherapy, dripping at 200-250 mL/hour for 4-6 hours. Generally, $3,000-4,000 \mathrm{~mL}$ of fluid will be administered on the same day, and $15 \mathrm{~mL}$ of potassium chloride will be supplemented for every $1,000 \mathrm{~mL}$ of fluid. No hydration will be required if cis-diamminedichloroplatinum (DDP) is used on a 4-day regimen.

\section{Timing for surgery}

Surgery will be performed 4-6 weeks after the last cycle of neoadjuvant therapy when the white blood cell (WBC) count, platelet count, and liver/kidney functions have returned to normal. Preoperative evaluations will include a routine blood test, blood biochemistry, chest and abdominal CT, pulmonary function testing, and an electrocardiogram (ECG). Bronchial endoscopy will be performed before surgery in patients with changes in tracheal or bronchial mucosa.

\section{Outcome measures}

\section{Pathological evaluation}

The pathological outcome measures include major pathologic response (MPR) and pCR.

\section{Radiological evaluation}

The radiological evaluation will be performed in terms of complete response (CR), partial response (PR), stable disease (SD), and progressive disease (PD).

The incidence of surgical complications will be recorded. Efficacy will be evaluated by barium swallow, chest and abdominal CT, and/or endoscopic ultrasound (EUS; a combination of ultrasound imaging and endoscopy to provides high-resolution, real-time imaging of the gastrointestinal tract and surrounding extramural structures) 2 weeks after the end of the immunotherapy combined with chemotherapy.

Bronchial endoscopy will be performed before immunotherapy plus chemotherapy in patients with changes in tracheal or bronchial mucosa.

\section{Response evaluation after immunotherapy plus chemotherapy}

(I) CR: the disappearance of all target lesions, as measured by barium swallow of the esophagus, the softness of the esophageal wall, the smooth passage of barium, no significant thickening of the esophageal wall on the CT scan of the chest, a wall thickness of $<5 \mathrm{~mm}$, the disappearance of enlarged lymph nodes, and no new lesions for up to 4 weeks or at least until the surgery;

(II) PR: at least 30\% decrease from baseline sum of the longest diameter, as demonstrated by barium swallow and chest CT, for up to 4 weeks or at least until the surgery;

(III) SD: reduction of all baseline target lesions not reaching $\mathrm{PR}$, or enlargement of the lesions not reaching $\mathrm{PD}$, as measured by barium swallow and chest CT;

(IV) PD: $\geq 20 \%$ increase in the sum of the longest diameters of all baseline target lesions, as measured by barium swallow and chest CT, or the appearance of one or more new lesions.

\section{Ethical standards}

\section{Informed consent}

Before a patient is enrolled in this study, the physician is obligated to provide the patient with a complete and comprehensive description of the purpose of the study and the properties of the drug and its possible toxicities and risks, and the patient should be made aware of their rights as well as the risks and benefits to be assumed. All subjects will be required to sign an informed consent form (https:// cdn.amegroups.cn/static/public/10.21037atm-21-6931-1. pdf), which will be kept in the case report form (CRF).

\section{Ethical standards, policies, and regulations}

This clinical trial complies with the Good Clinical Practice (GCP) issued by the State Food and Drug Administration 
(SFDA) of China, and related laws and regulations. Ethical approval has been approved by the local Ethics Committee of Taizhou Hospital (No. K20210822). Any change or modification to the clinical protocol must be reported to the ethics committee and recorded. The study will be conducted in accordance with the Declaration of Helsinki (as revised in 2013).

\section{Quality assurance}

All investigators and sponsors must conduct the trial in strict compliance with the research protocol, as required by the GCP. Efforts should be made to ensure standardized operating procedures, accurate test data, and reliable study conclusions.

\section{Requirements for coordinating investigators}

(I) Explain the clinical protocol to the investigators and give lectures on the completion of CRF before the clinical research is initiated;

(II) Send clinical research associates to conduct on-site supervision;

(III) Ensure smooth communication with the investigators at all times by telephone, fax, or email;

(IV) Provide center stratification and randomization services.

\section{Requirements for investigators}

(I) Obtain informed consent from each subject or the subject's legally authorized representative;

(II) Complete the $\mathrm{CRF}$ as required;

(III) Arrange regular visits;

(IV) Keep complete laboratory and clinical records as well as the original medical records of the subjects.

\section{Data processing and storage}

CRF: the CRF is to be completed by the investigators in a timely manner to ensure its accuracy and reliability. The CRF should generally not be modified. If a genuine error needs to be corrected, the correction should be signed (see instructions for completing the CRF). The CRF will be submitted in triplicate to the leader hospital, the sponsor, and the trial hospital, respectively, for archiving after the trial is completed. The clinical research associate will review the completed CRF before data entry. The content of the CRF should not be modified.

\section{Establishment of the database}

After receiving the CRF, the statistician will verify any doubtful points with the investigators, and the investigators should give feedback as soon as possible. The statistician will establish a database in a timely manner. After the database is reviewed, the data will be locked by the principal investigator, sponsor, statistician, and clinical research associate. Data security should be a top priority. Unauthorized access (or modification) of the data should be avoided. The data will be backed up securely.

\section{Storage of data}

The investigators should ensure the completeness of the data stored. According to the GCP principles in China, the data should be kept by the researcher for more than 5 years.

\section{Statistical analysis}

An intention to treat (ITT) analysis will be used, regardless of completion of the treatment.

\section{Calculation of survival data}

(I) Survival calculation: from the date of randomization to the date of death;

(II) Progression-free survival (PFS): from the date of randomization to the date of recurrence or metastasis;

(III) Survival rates: 3- and 5-year survival rates and relapseand metastasis-free survival rates will be calculated using the Kaplan-Meier method and compared statistically using the log-rank test;

(IV) ORR: the complete response plus the partial response;

(V) $\mathrm{P}$ values will be two-tailed, and the significance level will be set at $\mathrm{P}<0.05$.

Patients will be followed up by assigned research nurses outside the study group, who have also completed the registration and completion of CRFs. The data analysis will be conducted by the Design, Measurement and Evaluation in Clinical Research (DME) faculty.

\section{Follow-up}

A separate CRF will be created for each subject, and a research nurse will be employed to complete the followup, registration, form completion, and data maintenance. The subjects will be followed up regularly in the outpatient departments every 3 months for the first year after the end of treatment and then every 3-6 months until the patient's death or the end of the study. During each visit, the subject will receive a physical examination, chest $\mathrm{X}$-ray/barium swallow, abdominal ultrasound, or CT scans of the chest and abdomen. 
An esophagoscopy will be performed every 6 months.

Notably, subjects who withdraw from the study must also be followed up and included in the statistical analyses.

\section{Results}

\section{Study schedule}

(I) January 2022-December 2023: subject enrollment in Taizhou Hospital, Zhejiang Province ( $\mathrm{n}=50)$; Zhejiang Provincial Key Laboratory for Drug Evaluation and Clinical Research.

(II) January 2024-December 2025: subject randomization, follow-up, and data collection;

(III) 2023: interim report released, case randomization completed, and preliminary results reported, including the toxic effects of immunotherapy plus chemotherapy, patient tolerability, assessment of the impact of immunotherapy plus chemotherapy on prognosis, and 1- and 3-year DFS and OS;

(IV) 2026: the final study results will be published.

\section{Patient selection}

\section{The inclusion criteria}

(I) With informed consent forms signed by the patients;

(II) With confirmed resectable or BR ESCC;

(III) With advanced disease not previously treated systemically;

(IV) Aged 18-75 years;

(V) With an expected survival of $>6$ months;

(VI) WBC count $\geq 4.0 \times 10^{9} / \mathrm{L}$, neutrophils $\geq 1.5 \times 10^{9} / \mathrm{L}$, platelet count $\geq 100.0 \times 10^{9} / \mathrm{L}$, and hemoglobin $\geq 90 \mathrm{~g} / \mathrm{L}$, with normal liver and kidney functions;

(VII) With an Eastern Cooperative Oncology Group performance status (ECOG PS) score of $0-1$ point.

\section{Exclusion criteria}

(I) Has participated (or is currently participating) in any other antitumor clinical studies;

(II) Has received any previous treatment of esophageal cancer with systemic anticancer therapy as the primary treatment, including cytotoxic therapy, targeted therapy (including tyrosine kinase inhibitors or monoclonal antibodies), and immunotherapy, except for neoadjuvant use;

(III) Has received adjuvant therapy before enrollment;

(IV) With stage III-IV esophageal cancer and refusal to attend a clinical trial;

(V) Pregnant women or breastfeeding mothers;

(VI) Patients assessed by the investigators as unsuitable for enrollment, such as those with neurological or metabolic disorders, suspected of having illness on physical examination or laboratory tests, having contraindications to the use of the study drug, or at a high risk of treatment-related complications.

\section{Study endpoints}

\section{Primary endpoints}

The primary endpoints are DFS, MPR, and pCR.

\section{Secondary endpoints}

The secondary endpoints include ORR and overall survival (OS) in subjects with a PD-L1 expression level of $<1 \%$, $\geq 1 \%, \geq 20 \%$, and $\geq 50 \%$.

\section{Discussion}

ESCC responds poorly to conventional therapies such as surgery, chemotherapy, and radiotherapy. ESCC patients typically have a poor prognosis and low 5-year survival rates. Therefore, new treatment strategies are urgently needed to improve patients' OS and PFS. Emerging immunotherapy has achieved good efficacy for stage II and advanced esophageal cancer; however, whether it can replace the previous standard neoadjuvant chemotherapy needs to be further addressed.

The current research on esophageal cancer is mainly based on immunotherapy modalities such as antibody- and cell-based immune checkpoint blockers, pericyte therapy, and tumor vaccines. Immunotherapy, particularly immune checkpoint blockers, has brought new survival benefits to ESCC patients; in particular, immunotherapy has achieved satisfactory efficacy in patients with locally advanced ESCC. Chemotherapy, as a systemic adjuvant treatment for multiple tumors, can effectively kill tumor cells and control disease progression, while immunotherapy combined with chemotherapy can reverse immunosuppression in the advanced stages, improve cross-presentation of tumor antigens, promote proliferation of killer $\mathrm{T}$ cells (and increase their cancer-killing ability), and slow down the development of drug resistance of tumor cells. The combinations of immunotherapy with chemotherapy and surgery may provide new survival benefits for patients. Tislelizumab is a humanized IgG4 anti-PD-1 monoclonal 
antibody approved by the NMPA. Compared to other PD-1 inhibitors, tislelizumab has a higher affinity for PD-1 than pembrolizumab and nivolumab, which may be due to the difference in its PD-1 binding orientation. Tislelizumab is a humanized IgG4 monoclonal antibody, which can reduce the binding of Fc $\gamma$ Rs to macrophages so as to reduce antibody-dependent phagocytosis. Preclinical data show that tislelizumab does not bind to Fc $\gamma$ RI, while other anti-PD-1 antibodies bind to FcIgG4RI in a manner consistent with the affinity of human $\gamma$ antibodies. The treatment paradigm of immunotherapy combined with neoadjuvant chemotherapy has been explored in patients with locally advanced ESCC, and preoperative neoadjuvant immunotherapy combined with neoadjuvant chemotherapy has shown desirable results; however, there were no largesample multicenter studies.

In our project, a single-arm, multicenter, phase II clinical study of tislelizumab plus $\mathrm{ABP} /$ cisplatin as neoadjuvant therapy for BR ESCC will be performed. The role of preoperative concurrent immunotherapy plus chemotherapy in improving the survival rates of patients with BR ESCC will be explored, with an attempt to find a reasonable immunotherapy plus chemotherapy regimen with higher efficacy and lower toxicity, thus further increasing the pCR. We hope our study will identify a multidisciplinary treatment model for BR ESCC and provide evidence for the development of new guidelines on the diagnosis and treatment of esophageal cancer.

\section{Acknowledgments}

Funding: None.

\section{Footnote}

Reporting Checklist: The authors have completed the SPIRIT reporting checklist. Available at https://atm.amegroups. com/article/view/10.21037/atm-21-6931/rc

Conflicts of Interest: All authors have completed the ICMJE uniform disclosure form (available at https://atm. amegroups.com/article/view/10.21037/atm-21-6931/coif). The authors have no conflicts of interest to declare.

Ethical Statement: The authors are accountable for all aspects of the work in ensuring that questions related to the accuracy or integrity of any part of the work are appropriately investigated and resolved. The study will be conducted in accordance with the Declaration of Helsinki (as revised in 2013). Ethical approval has been approved by the local Ethics Committee of Taizhou Hospital (No. K20210822). Informed consent will be taken from all individual participants.

Open Access Statement: This is an Open Access article distributed in accordance with the Creative Commons Attribution-NonCommercial-NoDerivs 4.0 International License (CC BY-NC-ND 4.0), which permits the noncommercial replication and distribution of the article with the strict proviso that no changes or edits are made and the original work is properly cited (including links to both the formal publication through the relevant DOI and the license). See: https://creativecommons.org/licenses/by-nc-nd/4.0/.

\section{References}

1. Bray F, Ferlay J, Soerjomataram I, et al. Global cancer statistics 2018: GLOBOCAN estimates of incidence and mortality worldwide for 36 cancers in 185 countries. CA Cancer J Clin 2018;68:394-424.

2. Ferlay J, Soerjomataram I, Dikshit R, et al. Cancer incidence and mortality worldwide: sources, methods and major patterns in GLOBOCAN 2012. Int J Cancer 2015;136:E359-86.

3. Stahl M, Mariette C, Haustermans K, et al. Oesophageal cancer: ESMO Clinical Practice Guidelines for diagnosis, treatment and follow-up. Ann Oncol 2013;24 Suppl 6:vi51-6.

4. Vermorken JB, Mesia R, Rivera F, et al. Platinum-based chemotherapy plus cetuximab in head and neck cancer. $\mathrm{N}$ Engl J Med 2008;359:1116-27.

5. Hong MH, Kim HR, Park SY, et al. A phase II trial of preoperative chemoradiotherapy and pembrolizumab for locally advanced esophageal squamous cell carcinoma (ESCC). J Clin Oncol 2019;37:abstr 4027.

6. A Study of Tislelizumab (BGB-A317) in Combination With Chemotherapy as First Line Treatment in Participants With Advanced Esophageal Squamous Cell Carcinoma. Available online: https://clinicaltrials.gov/ct2/ show/NCT03783442

7. International Agency for Research on Cancer in 2018, WHO data cancer today. Available online: https://gco.iarc. fr/today/explore

8. Kudo T, Hamamoto Y, Kato K, et al. Nivolumab treatment for oesophageal squamous-cell carcinoma: an open-label, multicentre, phase 2 trial. Lancet Oncol 2017;18:631-9. 
9. Kato K, Cho BC, Takahashi M, et al. Nivolumab versus chemotherapy in patients with advanced oesophageal squamous cell carcinoma refractory or intolerant to previous chemotherapy (ATTRACTION-3): a multicentre, randomised, open-label, phase 3 trial. Lancet Oncol 2019;20:1506-17.

10. Shah MA, Kojima T, Hochhauser D, et al. Efficacy and Safety of Pembrolizumab for Heavily Pretreated Patients With Advanced, Metastatic Adenocarcinoma or Squamous Cell Carcinoma of the Esophagus: The Phase 2 KEYNOTE-180 Study. JAMA Oncol 2019;5:546-50.

11. Kojima T, Shah MA, Muro K, et al. Randomized Phase III KEYNOTE-181 Study of Pembrolizumab Versus Chemotherapy in Advanced Esophageal Cancer. J Clin Oncol 2020;38:4138-48.

12. Metges J, Francois E, Shah M, et al. The phase 3 KEYNOTE-181 study: pembrolizumab versus chemotherapy as second-line therapy for advanced esophageal cancer. Ann Oncol 2019;30:abstr iv130.

13. Kato K, Shah MA, Enzinger P, et al. KEYNOTE-590: Phase III study of first-line chemotherapy with or without pembrolizumab for advanced esophageal cancer. Future Oncol 2019;15:1057-66.

14. Kelly RJ, Lockhart AC, Jonker DJ, et al. CheckMate 577: A randomized, double-blind, phase 3 study of adjuvant nivolumab (nivo) or placebo in pts with resected esophageal (E) or gastroesophageal junction (GEJ) cancer. J Clin Oncol 2017;35:abstr TPS4131.

15. Ajani JA, Kato K, Doki Y, et al. CheckMate 648: A

Cite this article as: $\mathrm{Li} \mathrm{X}, \mathrm{Xu} C$, Qiu $\mathrm{H}$, Chen $\mathrm{D}$, Zhu K, Zhang B, Zhang J, Xu A, Wang C, Shen J. A single-arm, multicenter, phase II clinical study of tislelizumab plus albuminbound paclitaxel/cisplatin as neoadjuvant therapy for borderline resectable esophageal squamous cell carcinoma. Ann Transl Med 2022;10(5):263. doi: 10.21037/atm-21-6931 randomized phase 3 study of nivolumab plus ipilimumab or nivolumab combined with fluorouracil plus cisplatin versus fluorouracil plus cisplatin in patients with unresectable advanced, recurrent, or metastatic previously untreated esophageal squamous cell carcinoma. J Clin Oncol 2018;36:abstr TPS193.

16. Huang J, Xu J, Chen Y, et al. Camrelizumab versus investigator's choice of chemotherapy as second-line therapy for advanced or metastatic oesophageal squamous cell carcinoma (ESCORT): a multicentre, randomised, open-label, phase 3 study. Lancet Oncol 2020;21:832-42.

17. Guidelines Working Committee of Chinese Society of Clinical Oncology. Guidelines for Diagnosis and Treatment of Esophageal Cancer (2020 Edition). Beijing: People's Medical Publishing House, 2020.

18. Chao J, Fuchs CS, Shitara K, et al. Assessment of Pembrolizumab Therapy for the Treatment of Microsatellite Instability-High Gastric or Gastroesophageal Junction Cancer Among Patients in the KEYNOTE-059, KEYNOTE-061, and KEYNOTE-062 Clinical Trials. JAMA Oncol 2021;7:895-902.

19. van den Ende T, de Clercq NC, van Berge Henegouwen MI, et al. Neoadjuvant Chemoradiotherapy Combined with Atezolizumab for Resectable Esophageal Adenocarcinoma: A Single-arm Phase II Feasibility Trial (PERFECT). Clin Cancer Res 2021;27:3351-9.

(English Language Editor: D. Fitzgerald) 